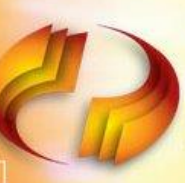

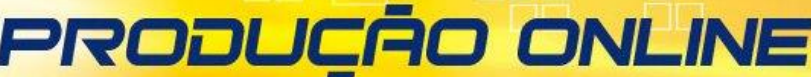 REVISTA CIENTIFICA ELETRO̊NICA DE ENGENHARIA DE PRODUÇÃO ISSN 1676-1901
}

\section{GERENCIAMENTO DE RISCOS PARA A CADEIA DE SUPRIMENTOS: UMA REVISÃO SISTEMÁTICA DE LITERATURA}

\section{SUPPLY CHAIN RISKS MANAGEMENT: A SYSTEMATIC LITERATURE REVIEW}

\author{
Matheus Borges Carneiro* E-mail: matheusborgescarneiro@outlook.com \\ Flávio Henrique de Oliveira Costa* E-mail: flaviohenrique@facef.br \\ Thalisa Maria Jati Gilberto* E-mail: thalisa@facef.br \\ Robson Nogueira Tomas ${ }^{\star *}$ E-mail: robson.tomas@ifsuldeminas.edu.br \\ ${ }^{*}$ Centro Universitário Municipal de Franca (Uni-FACEF), Franca, SP \\ **Instituto Federal de Educação, Ciência e Tecnologia do Sul de Minas Gerais (IFSULDEMINAS), Pouso \\ Alegre, MG
}

Resumo: Os debates acerca da gestão de riscos para a cadeia de suprimentos têm crescido nos últimos anos, tanto em âmbito internacional quanto nacional, devido ao impacto dos riscos no desempenho das empresas. Assim, este artigo tem por objetivo apresentar os principais conceitos relacionados ao gerenciamento de riscos em cadeias de suprimentos, os modelos para a gestão destes riscos, bem como estratégias para mitiga-los. Para tanto, foi feita uma revisão sistemática de literatura para identificar as principais pesquisas nesse sentido, nas áreas de gestão de riscos voltada principalmente aos riscos no fornecimento e na demanda, bem como na busca por técnicas e estratégias para mitigação dos riscos. Para trabalhos futuros, sugerem-se a busca por técnicas para medição do desempenho das estratégias de mitigação propostas, assim como uma análise em uma empresa ou cadeia de valor, a fim de se propor um modelo eficaz de gerenciamento dos riscos.

Palavras-chave: Gestão de riscos. Gerenciamento de riscos em cadeias de suprimentos. Revisão sistemática de literatura. Riscos no fornecimento e na demanda. Estratégias para mitigação dos riscos.

Abstract: Debates about supply chain risk management have increased in recent years, both internationally and nationally, due to the risks impact on business performance. Therefore, this article aims to present the main concepts related to supply chain risk management, risk management models, as well as strategies to mitigate them. To achieve so, a systematic literature review was conducted to identify the main research in this regard, in the areas of risk management focused mainly on supply and demand risks, as well as in the search for risk mitigation strategies and techniques. For future work, it is suggested a search for techniques for performance measurement of the proposed mitigation strategies, such as an analysis on a company or value chain, in order to propose and effective risk management model.

Keywords: Risks management. Supply chain risks management. Systematic literature review. supply and demand risks. Risks mitigation strategies.

\section{INTRODUÇÃO}

O aumento do nível competitivo, demanda das empresas a adoção de práticas como lean manufacturing, filosofia JIT (just in time), omnichannel, terceirização, redução da base de suprimentos, técnicas alternativas para gestão de estoques, entre 
outras, que visam otimizar processos, eliminar desperdícios, reduzir gastos e satisfazer a demanda, envolvendo, contudo, um nível considerável de riscos tanto à empresa, quanto à toda a cadeia de valor, devido ao fato de reduzirem os níveis de estoques e, consequentemente, tornarem toda a cadeia suscetível aos riscos (BANDALY et al., 2012; ENYINDA, 2018; VISHNU et al., 2019).

Os debates acerca dos riscos à cadeia de suprimentos vêm sendo discutido em âmbito internacional, devido aos inúmeros atentados terroristas (JUTTTNER et al., 2003; FINCH, 2004), acidentes naturais (VILKO; HALLIKAS, 2012) e crises econômicas (MANUJ; MENTZER, 2008). Além destes riscos, há ainda as falhas da comunicação entre os elos, que pode ocasionar o efeito-chicote (VAN LANDEGHEM; VANMAELE, 2002).

Por sua vez, o Brasil se encontra submetido à inúmeros riscos, como a greve dos caminhoneiros de maio de 2018 (FOLETTO, 2018), o vazamento de óleo na Bacia dos Campos, em 2011 (RIBEIRO, 2011), o incêndio no terminal portuário de Santos, em 2015 (VENTURA, 2015), o rompimento das barragens em Miraí, em 2007, em Mariana, em 2015 (GONÇALVES, 2017) e em Brumadinho, em 2019 (SENRA, 2019).

Mesmo em um cenário instável e suscetível a inúmeros riscos, o debate acerca do gerenciamento de riscos no Brasil é recente, com Tomas e Alcantara (2013) apresentando estruturas para gerenciamento de riscos. Camargo Junior et al. (2014) apresentam a coopetição como estratégia para mitigação dos riscos, uma vez que esta técnica combina os conceitos de cooperação e competição entre concorrentes, e fortalece alianças estratégicas para mitigar riscos mútuos. Oliveira et al. (2015) analisam os riscos na cadeia agroindustrial de frango. Por fim, Silva et al. (2017) desenvolveram uma estrutura para gerenciamento dos riscos em duas empresas do setor alimentício.

Nesse sentido, a presente pesquisa tem por objetivo apresentar os principais conceitos relacionados ao gerenciamento de riscos em cadeias de suprimentos, bem como apresentar os principais modelos para sua gestão eficaz. Logo, este artigo busca contribuir para futuras pesquisas teóricas e empíricas relacionadas ao tema, de modo que sua relevância se dá na escassez de trabalhos nacionais com o tema em questão. 
Este trabalho encontra-se estruturado da seguinte maneira. A seção 2 apresenta os procedimentos metodológicos e detalha a revisão sistemática de literatura realizada. A Seção 3 expõem o processo de identificação dos riscos para a cadeia de suprimentos, enquanto a seção 4 apresenta os principais frameworks para geri-los. A Seção 5 discute os fatores-chave identificados através da pesquisa e apresenta estratégias de mitigação dos riscos. Por fim, a seção 6 destaca os resultados alcançados a partir do objetivo do trabalho em questão, e propõem perspectivas para estudos futuros.

\section{MÉTODO DE PESQUISA}

Fawccett et al.. (2014) mencionam que, devido a evolução da internet e ao frequente surgimento de novos congressos e periódicos, há uma vasta quantidade de informação disponível, de modo que, para se desenvolver uma revisão de literatura consistente e essencial a um trabalho científico adequado, se faz cada vez mais necessário adotar a abordagem da revisão sistemática de literatura (ou Systematic Literature Review).

Colicchia et al. (2012) definem a SLR como uma metodologia analítica para identificar, selecionar, analisar e avaliar criticamente pesquisas relevantes, de modo a coletar e interpretar os dados incluídos na revisão. Para Tomas e Alcantara (2013), este método proporciona maior confiabilidade a pesquisa, e pode identificar lacunas na literatura a serem exploradas.

Para a utilização deste método, levou-se em consideração a estrutura sugerida por Tranfield, Denyer e Smart (2003), que apontam três estágios para o desenvolvimento da revisão sistemática da literatura, sendo eles: I - planejamento da revisão (que engloba a definição das questões e do protocolo de pesquisa); II condução da sistemática (que envolve a busca dos artigos, a aplicação dos filtros e a leitura analítica dos mesmos); III - disseminação do estudo (a exposição dos resultados da leitura analítica dos artigos).

A primeira etapa foi a leitura de escopo. Nesta etapa, foram lidos trabalhos como Juttner, Peck e Christopher (2003); Tomas, Alcantara (2013); Tomas (2014) e Oliveira, Espindola e Marins (2017) a fim de delimitar o tema e definir as strings. A 
segunda etapa foi a definição das questões a serem respondidas através da revisão sistemática, que foram:

a.) Como identificar os riscos na cadeia de suprimentos?

b.) Quais são os modelos estratégicos para o gerenciamento dos riscos na cadeia de suprimentos?

É possível compreender as principais etapas da revisão sistemática de literatura a partir do protocolo desenvolvido, conforme pode ser observado na Tabela 1, apresentado a seguir.

Tabela 1 - Protocolo da revisão sistemática

\begin{tabular}{|c|c|c|}
\hline Estágio & Obtenção & Detalhes \\
\hline $\begin{array}{l}\text { Leitura de } \\
\text { Escopo }\end{array}$ & $\begin{array}{c}\text { Busca nos principais periódicos na área } \\
\text { de SCM, Operações e Sustentabilidade } \\
\text { citados em trabalhos no tema e indicação } \\
\text { de especialistas. }\end{array}$ & $\begin{array}{l}\text { * Definir o problema de pesquisa. } \\
\text { * Identificar constructos. } \\
\text { * Encontrar palavras-chave (para strings). }\end{array}$ \\
\hline $\begin{array}{l}\text { Condução } \\
\text { da Revisão }\end{array}$ & $\begin{array}{l}\text { Buscas nas bases de dados: ISI Web of } \\
\text { Science, SciELO e Spell, no intervalo de } \\
\text { tempo de } 2000 \text { a } 2018\end{array}$ & $\begin{array}{c}\text { 1ํ Filtro: leitura do título e abstract. } \\
\text { 2º Filtro: Leitura da Introdução e da conclusão. } \\
\text { Analisar acesso e qualidade do periódico. } \\
\text { 3ํ Filtro/Classificação: Leitura completa e } \\
\text { avaliação da qualidade do artigo (leitura } \\
\text { analítica). }\end{array}$ \\
\hline $\begin{array}{c}\text { Análise de } \\
\text { dados }\end{array}$ & Leitura dos artigos selecionados & Ler e analisar o material selecionado. \\
\hline
\end{tabular}

Fonte: Elaborado pelos autores (2019)

Para realizar a busca de artigos nas bases mencionadas, foram definidas as strings de busca, assim como os constructos e as palavras-chave. O desenvolvimento das palavras-chave foi feito com base na leitura de escopo. Como as pesquisas acerca de gestão de riscos na cadeia de suprimentos é recente, optou-se por artigos entre 2000 e 2018. Além disso, para agregar maior valor à revisão sistemática, foram desconsiderados os artigos de congressos (proceeding papers), resenhas de artigos, e editoriais. A Tabela 2 apresenta os constructos utilizados para esta pesquisa. 
Tabela 2 - Constructos da pesquisa

\begin{tabular}{|c|c|c|c|}
\hline Questão respondida & Constructos & Palavras- chave & String \\
\hline $\begin{array}{l}\text { 1. Como identificar os } \\
\text { riscos de suprimento e } \\
\text { demanda? }\end{array}$ & $\begin{array}{c}\text { Gestão de } \\
\text { riscos na cadeia } \\
\text { de suprimentos }\end{array}$ & $\begin{array}{l}\text { * Supply chain risks } \\
\text { management; } \\
\text { * Supply chain risk } \\
\text { management; } \\
\text { * Supply risks; } \\
\text { * Demand risks; } \\
\text { * Risk management }\end{array}$ & $\begin{array}{c}\text { (supply chain AND (("risk } \\
\text { management") OR ("demand } \\
\text { risks*") OR ("supply risks*") OR } \\
\text { ("risk" management)) }\end{array}$ \\
\hline $\begin{array}{l}\text { 2. Quais são os } \\
\text { modelos estratégicos } \\
\text { para o gerenciamento } \\
\text { dos riscos na cadeia } \\
\text { de suprimentos? }\end{array}$ & $\begin{array}{l}\text { Mitigação de } \\
\text { riscos. } \\
\text { Gestão de } \\
\text { riscos na cadeia } \\
\text { de suprimentos. }\end{array}$ & $\begin{array}{l}\text { * Risk Assessment; } \\
\text { * Risk sources; } \\
\quad{ }^{*} \text { Risk drivers; } \\
\text { * Risk consequences; }\end{array}$ & $\begin{array}{c}\text { (("Risk Assessment"") OR ("risk } \\
\text { sources") OR ("risk drivers") OR } \\
\text { ("risk consequences") OR ("risk } \\
\text { mitigation") OR ("strategies for risk } \\
\text { mitigation") OR ("risk mitigating } \\
\text { strategies")) }\end{array}$ \\
\hline
\end{tabular}

Fonte: Elaborado pelos autores (2019)

Para desenvolvimento da revisão sistemática de literatura, foram adotadas as bases: ISI Web of Science, SciELO e SPELL. Para a realização da revisão sistemática, foram adotados três filtros, conforme foi observado na Tabela 1. A Tabela 3, apresentada a seguir, aponta quais os critérios para inclusão ou exclusão dos artigos, para cada filtro proposto.

Tabela 3 - Filtros de pesquisa

\begin{tabular}{|c|c|c|c|}
\hline Filtro & Critério & Inclusão & Exclusão \\
\hline & $\begin{array}{l}\text { Gerenciamento de } \\
\text { riscos na cadeia } \\
\text { de suprimentos }\end{array}$ & $\begin{array}{c}\text { Tratar diretamente a respeito da } \\
\text { gestão de riscos para a cadeia } \\
\text { de suprimentos. }\end{array}$ & $\begin{array}{l}\text { Não se referir diretamente à gestão } \\
\text { de riscos na cadeia de suprimentos. }\end{array}$ \\
\hline 1 & $\begin{array}{l}\text { Modelos de } \\
\text { mitigação dos } \\
\text { riscos }\end{array}$ & $\begin{array}{l}\text { Propor estratégias para a } \\
\text { identificação, avaliação ou } \\
\text { mitigação dos riscos suscetíveis } \\
\text { à cadeia de suprimentos }\end{array}$ & $\begin{array}{l}\text { Não propor modelos, técnicas ou } \\
\text { estratégias para identificar, avaliar } \\
\text { ou mitigar os riscos da cadeia de } \\
\text { suprimentos. }\end{array}$ \\
\hline \multirow[t]{3}{*}{2} & Acesso & $\begin{array}{l}\text { Possuir acesso ao trabalho, estar } \\
\text { redigido em inglês, espanhol ou } \\
\text { português. }\end{array}$ & $\begin{array}{l}\text { Não possuir acesso ao trabalho, não } \\
\text { estar redigido em inglês, espanhol } \\
\text { ou português. }\end{array}$ \\
\hline & Qualidade & $\begin{array}{c}\text { Periódico cientifico com revisão } \\
\text { de pares }\end{array}$ & $\begin{array}{c}\text { Periódico cientifico sem revisão de } \\
\text { pares, jornais de negócios, revistas } \\
\text { correntes, conferências, livros e } \\
\text { sites. }\end{array}$ \\
\hline & Temática & $\begin{array}{l}\text { Conceitos de gestão de riscos na } \\
\text { cadeia de suprimentos, ou } \\
\text { modelos de gerenciamento de } \\
\text { riscos para empresas focais, } \\
\text { voltados aos riscos da } \\
\text { demanda/suprimentos }\end{array}$ & $\begin{array}{l}\text { Conceitos referentes a ciência dos } \\
\text { materiais ou do meio ambiente, } \\
\text { fisiologia, psicologia e } \\
\text { comportamento humano, saúde e } \\
\text { estudos urbanísticos, ou riscos para } \\
\text { gestão de projetos }\end{array}$ \\
\hline
\end{tabular}

Revista Produção Online. Florianópolis, SC, v. 19, n. 3, p. 1048-1068, 2019 
Fonte: Elaborado pelos autores, 2019.

Os resultados da revisão sistemática e cada filtro pode ser observado na Figura

1, apresentada a seguir:

Figura 1 - Filtros aplicados à revisão sistemática de literatura

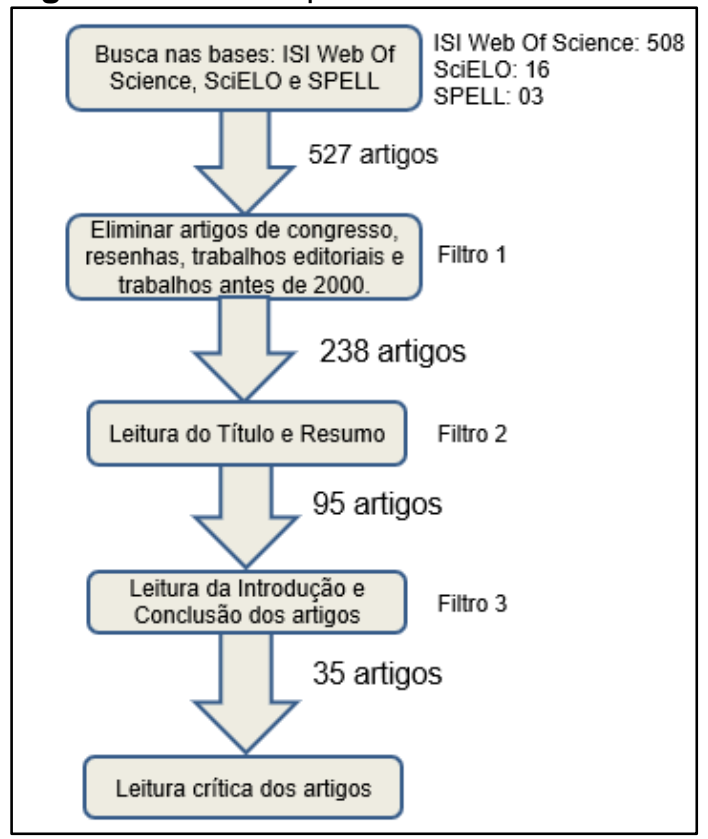

Fonte: Elaborado pelos autores (2019)

Após a execução dos filtros, foi possível analisar os artigos compostos nesta revisão sistemática. A Tabela 4 apresenta a quantidade de artigos por periódico.

Tabela 4 - Artigos por periódico

\begin{tabular}{cc}
\hline Periódico & Artigos \\
\hline $\begin{array}{c}\text { Supply Chain Management - An International Journal } \\
\text { International Journal of Production Research } \\
\text { Journal of Business Logistics }\end{array}$ & 4 \\
Produção Online & 2 \\
International Journal of Physical Distribution \& Logistics Management & 2 \\
Benchmarking- An International Journal & 2 \\
European Journal of Industrial Engineering & 1 \\
European Journal of Operational Research & 1 \\
Gestão \& Produção & 1 \\
Interfaces & 1 \\
International Journal of Logistics & 1 \\
International Journal of Logistics-Research and Applications & 1 \\
International Journal of Operations \& Production Management & 1 \\
International Journal of Quality \& Reliability Management & 1 \\
Journal of Advanced Manufacturing Systems & 1 \\
Journal of Manufacturing Technology Management & 1 \\
Journal of Operations Management & 1 \\
Journal of Purchasing and Supply Management & 1 \\
Journal of Risk Research & 1 \\
\hline
\end{tabular}

Revista Produção Online. Florianópolis, SC, v. 19, n. 3, p. 1048-1068, 2019 


\begin{tabular}{cc}
\hline Journal of Transport and Supply Chain Management & 1 \\
Operations and Supply Chain Management - An International Journal & 1 \\
Production and Operations Management & 1 \\
Production Planning \& Control & 1 \\
Revista de Administração Faces & 1 \\
Risk Management - An International Journal & 1 \\
Total & 35 \\
\hline
\end{tabular}

Fonte: Elaborado pelos autores (2019)

Logo, é possível observar que apenas quatro dos artigos identificados se referem a periódicos nacionais, o que evidencia a escassez de debates acerca deste tema no Brasil. A Figura 2 apresenta o gráfico com a quantidade de artigos selecionados na revisão por ano.

Figura 2 - Quantidade de artigos por ano

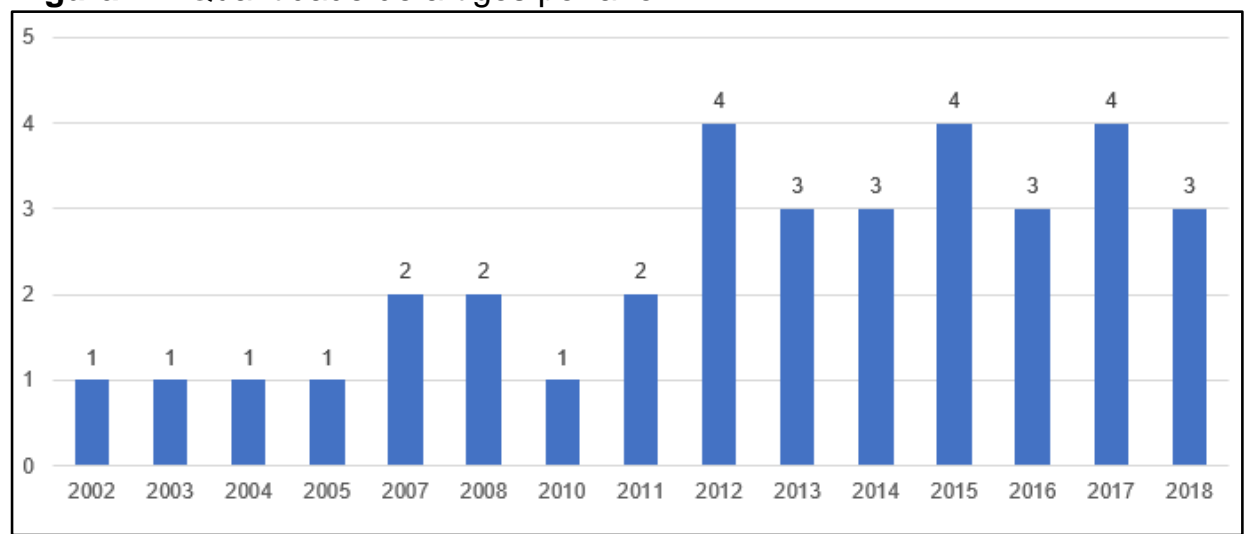

Fonte: Elaborado pelos autores (2019)

Como pode-se observar na Figura 2, há uma concentração maior de pesquisas relacionas à gestão de riscos na cadeia de suprimentos a partir de 2012, o que foi observado por Matos et al. (2017) e Oliveira et al. (2017).

\section{GESTÃO DE RISCOS PARA A CADEIA DE SUPRIMENTOS}

Fawccett et al. (2014) mencionam que, devido a evolução da internet e ao frequente surgimento de novos congressos e periódicos, há uma vasta quantidade de informação disponível, de modo que, para se desenvolver uma revisão de literatura consistente e essencial a um trabalho científico adequado, se faz cada vez mais necessário adotar a abordagem da revisão sistemática de literatura (RSL).

Para compreender o impacto dos riscos na cadeia de suprimentos, deve-se primeiramente compreender os riscos no segmento empresarial. Desse modo, o risco é o fator que pode impedir as atividades de desempenharem seu papel. Manuj e 
Mentzer (2008) e Vilko e Hallikas (2012) descreveram uma fórmula simples para estimar os riscos, descrita como:

$$
\text { Risco }=\text { Probabilidade * Impacto (1) }
$$

Nesse sentido, Sheffi e Rice (2005) apresentaram uma matriz 2x2 para apresentar o grau de vulnerabilidade em relação aos riscos, conforme apresentado na Tabela 5.

Tabela 5 - Matriz da vulnerabilidade de Sheffi e Rice (2005)

\begin{tabular}{lcc} 
& Baixo Impacto & Alto impacto \\
\hline Alta probabilidade de riscos ou ruptura & & Alta vulnerabilidade \\
Baixa probabilidade de riscos ou ruptura & Baixa vulnerabilidade & \\
\hline
\end{tabular}

Fonte: Adaptado de Sheffi; Rice (2005)

Para cada quadrante da matriz, sendo eles (SHEFFI; RICE, 2005; MONROE et al.., 2014):

- Alta probabilidade e Baixo impacto: problemas com transporte;

- Alta probabilidade e Alto impacto: perda de principal fornecedor, problemas com qualidade, ou recessão econômica;

- Baixa probabilidade e Baixo impacto: violência no local de trabalho;

- Baixa probabilidade e Alto impacto: terrorismo, terremotos, ou falência de fornecedores.

Assim, Wagner e Bode (2008) definem o risco como o desvio negativo do valor esperado de uma determinada medida de desempenho, resultando em consequências indesejáveis para a empresa focal. A ISO 31000:2018 (ABNT, 2018), que trata da gestão de riscos, complementa esta visão, ao afirmar que o risco pode ser um desvio, negativo ou positivo, em relação ao esperado, e pode abordar, criar ou resultar em oportunidades e ameaças. Por sua vez, Manuj e Mentzer (2008) apresentam três dimensões dos riscos: o impacto das perdas; a frequência que ocorrem; a velocidade com que ocorrem e com que podem ser identificados.

Nesse cenário de riscos empresariais, se insere a cadeia de suprimentos, definida por Chopra e Meindl (2003) como todos os elos envolvidos no atendimento do pedido do cliente, que parte desde os fornecedores primários, as fabricantes, aos varejistas e atacadistas, incluindo as transportadoras e os depósitos de armazenagem. Pires (2016) destaca o benefício competitivo da gestão das cadeias 
de suprimentos, que podem agregar valor ao cliente final e desenvolver vantagem competitiva a todos os elos da cadeia. Contudo, Aguiar (2010) comenta que as cadeias de suprimentos são suscetíveis aos riscos. Por conseguinte, Aguiar (2010) desenvolveu uma área de intersecção entre a gestão da cadeia de suprimentos e a gestão de riscos, o que resulta na gestão de riscos à cadeia de suprimentos (do inglês "Supply Chain Risks Management" ou SCRM), conforme apresentado na Figura 3.

Figura 3 - Área de interseção resultante no SCRM

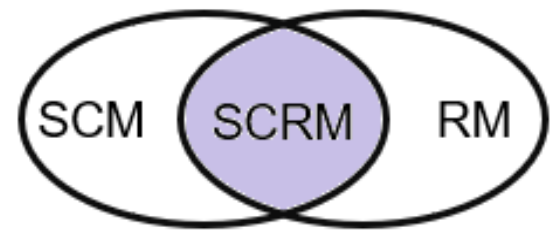

Fonte: Adaptado de Aguiar (2010) e Tomas; Alcantara (2013)

Silva et al. (2017) comentam que as cadeias de suprimentos são vulneráveis por fatores internos, como falta de compartilhamento de informações, ou externos, como questões sociopolíticas, ambientais ou econômicas. Christopher e Lee (2004) apontam que as principais causas da vulnerabilidade na cadeia estão atribuídas aos eventos externos, como guerras ou atentados terroristas, e decisões estratégicas empresariais, como práticas enxutas, aumento de decisões de terceirização, iniciativas para reduzir base de fornecedores.

Completando esta visão, Tang e Tomlin (2008) apresentam o aumento da variedade de produção, constante desenvolvimento de novos produtos e aumento do número de canais de distribuição e vendas como fatores que corroboram à vulnerabilidade. Monroe et al. (2014) ressaltam que a presença de vulnerabilidade na cadeia corrobora para a ruptura, de modo que possa gerar riscos para todos os elos envolvidos. Devido ao fato de a ruptura impactar no desempenho financeiro das empresas, Wagner e Neshat (2010) ressaltam a importância do estudo da gestão de riscos à cadeia de suprimentos se torna necessário.

Vishnu et al. (2019) apontam que, para a cadeia de suprimentos, os riscos se referem a quaisquer fatores que resultem em interrupções no fluxo de materiais, informações e recursos financeiros na cadeia que resultem em consequências indesejáveis e vulnerabilidade, gerando uma incompatibilidade entre suprimentos e 
demanda, de modo a afetar a eficiência e funcionamento da cadeia, podendo ainda conduzir às empresas a falência.

Desse modo, Funo et al. (2013) mencionam que a adoção do SCRM traz como benefícios a integração na cadeia para promover vantagem competitiva, redução de custos e alinhamento nas decisões estratégicas. Para Oliveira et al. (2018), a implantação do SCRM visa reduzir a frequência ou o impacto dos riscos na cadeia de suprimentos. Nesse quesito, as principais definições da SCRM obtidas a partir da RSL encontram-se na Tabela 6.

Tabela 6 - Definição de SCRM

\begin{tabular}{|c|c|}
\hline Definição & Autores \\
\hline $\begin{array}{l}\text { É a identificação e o gerenciamento dos riscos em uma cadeia de suprimentos a } \\
\text { partir de uma abordagem coordenada entre os elos da cadeia, visando diminuir a } \\
\text { vulnerabilidade da cadeia. }\end{array}$ & $\begin{array}{c}\text { Juttner et al. } \\
(2003)\end{array}$ \\
\hline $\begin{array}{l}\text { É o processo de mitigar os riscos a partir da colaboração coordenada e aplicação } \\
\text { das ferramentas de gestão de riscos entre os parceiros, para assegurar a } \\
\text { continuidade aliada à lucratividade a longo prazo da cadeia de suprimentos }\end{array}$ & $\begin{array}{l}\text { Faisal et al.. } \\
\text { (2007) }\end{array}$ \\
\hline $\begin{array}{l}\text { É um programa que inclui o processo de identificar o risco, quantifica-lo, atribuir } \\
\text { responsabilidades para o gerenciamento do risco, assim como ações para } \\
\text { mitigação. }\end{array}$ & $\begin{array}{l}\text { Handfield e } \\
\text { McCormack } \\
\text { (2008) }\end{array}$ \\
\hline $\begin{array}{l}\text { É a identificação e avaliação dos riscos e perdas para toda a cadeia, bem como } \\
\text { a implantação de estratégias apropriadas a partir de uma abordagem coordenada } \\
\text { entre os elos da cadeia com o objetivo de reduzir perdas e riscos, para os } \\
\text { resultados da cadeia serem o mais próximo do esperado. }\end{array}$ & $\begin{array}{c}\text { Manuj e } \\
\text { Mentzer (2008) }\end{array}$ \\
\hline $\begin{array}{l}\text { É a identificação do potencial de risco e implementação de estratégias } \\
\text { apropriadas através de uma abordagem coordenada entre os elos da cadeia de } \\
\text { suprimentos, de modo a reduzir a vulnerabilidade da cadeia. }\end{array}$ & $\begin{array}{l}\text { Monroe et al. } \\
(2014)\end{array}$ \\
\hline $\begin{array}{l}\text { É a combinação da probabilidade de um acontecimento e de suas consequências } \\
\text { para o negócio, podendo ser benéfica ou não ao negócio, dependendo de seu } \\
\text { resultado. }\end{array}$ & $\begin{array}{l}\text { Camargo Júnior } \\
\text { et al. (2014) }\end{array}$ \\
\hline $\begin{array}{l}\text { Um processo de várias etapas consistindo em avaliar a origem dos riscos, definir } \\
\text { o conceito dos riscos, identificar os direcionadores dos riscos e, enfim, mitigar os } \\
\text { riscos. }\end{array}$ & $\begin{array}{l}\text { Rogers et al. } \\
\quad(2015)\end{array}$ \\
\hline $\begin{array}{l}\text { Dividido em quatro etapas - identificação, avaliação, mitigação e monitoramento } \\
- \text {, tem como objetivo reduzir a frequência e/ou o impacto dos riscos nas cadeias } \\
\text { de suprimentos }\end{array}$ & $\begin{array}{l}\text { Oliveira et al. } \\
\qquad(2018)\end{array}$ \\
\hline $\begin{array}{l}\text { É uma área do estudo da gestão da cadeia de suprimentos, que aborda a gestão } \\
\text { eficaz de rupturas e vulnerabilidades na cadeia, determinando a capacidade de } \\
\text { uma cadeia de suprimentos sincronizar demanda e suprimentos, através de um } \\
\text { processo de várias etapas. }\end{array}$ & $\begin{array}{l}\text { Vishnu et al. } \\
\text { (2019) }\end{array}$ \\
\hline
\end{tabular}

Fonte: Dados da pesquisa (2019)

Prakash et al. (2017) afirmam que a etapa de identificação de riscos deve mapear todas as possíveis causas ou origens dos riscos para a cadeia. Os riscos na cadeia de suprimentos devem ser analisados em suas distintas categorias, separados por Manuj e Mentzer (2008) entre fornecimento, operacional e demanda, no qual cada 
elo conta com seus riscos individuais que impactam toda a cadeia, conforme Figura 4, apresentada a seguir.

Figura 4- Principais riscos nas categorias da cadeia

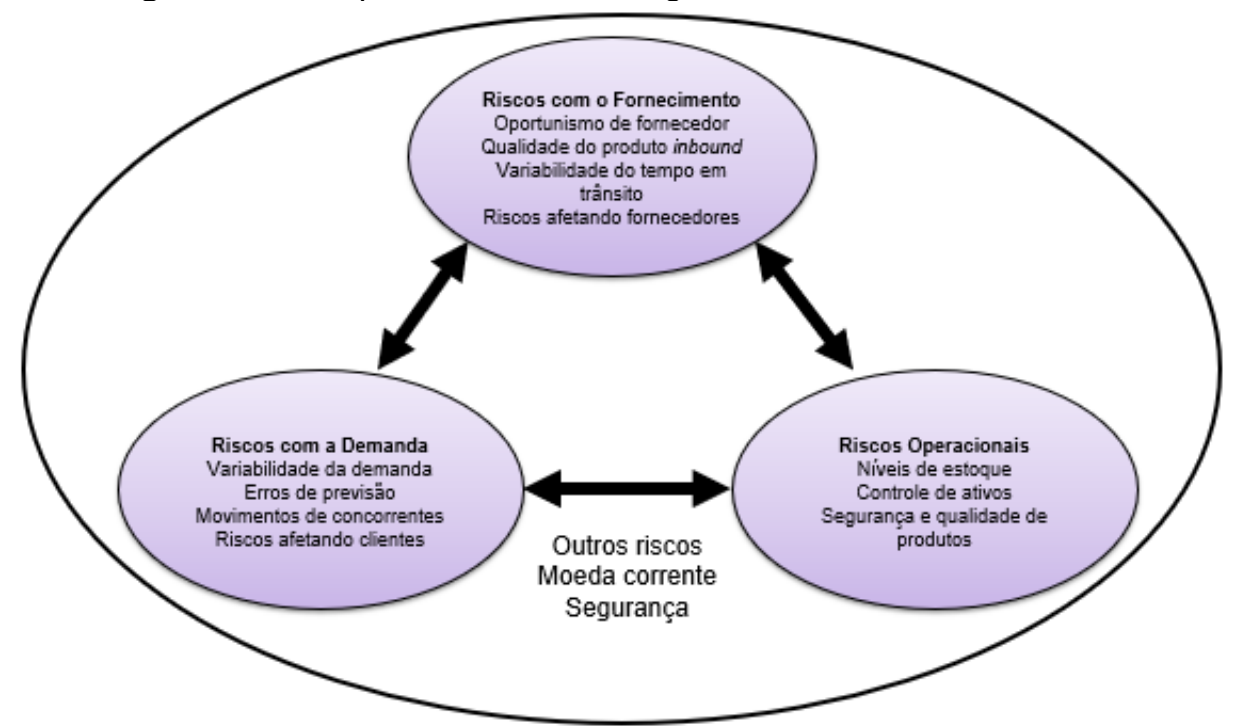

Fonte: Adaptado de Manuj; Mentzer (2008)

A Figura 4 define que os riscos relacionados a cadeia de suprimentos podem se referir a riscos com a demanda, com o fornecimento e operacionais. A conexão entre os riscos demonstra a interferência de um risco em outro, como os erros de previsão podem ocasionar falta de estoque. Os demais riscos se referem a possibilidades externas à cadeia, como alteração na moeda corrente e segurança do fluxo das informações e dos materiais entre os elos da cadeia.

Através da RSL, foi possível encontrar outras abordagens para a identificação dos riscos na cadeia, que cabem ser aqui mencionadas. Para Christopher e Peck (2004), os riscos podem ser internos à empresa focal - relacionado aos processos operacionais e ao planejamento da empresa -, internos à cadeia - relacionados a demanda e ao fornecimento - e externos a cadeia, se referindo a ataques terroristas, acidentes naturais, entre outros aspectos. De modo similar, Diabat et al. (2012) afirmam que os riscos podem ser subdivididos em cinco categorias: I- riscos com fornecimento; II- riscos com a demanda; III- riscos com o fluxo de informações; IVriscos com o produto/serviço; V- riscos macro, externos à cadeia. Como nenhum dos trabalhos nacionais desenvolveu o processo de identificação dos riscos pelo modelo 
de Manuj e Mentzer (2008), a presente pesquisa preenche esta lacuna ao adotar sua estrutura para identificação e categorização dos riscos.

Os riscos podem ser identificados através de dois fatores: as percepções dos gerentes - que definem o risco baseado especificamente na empresa e cadeia que atuam - (JUTTNER et al.; 2003; BANDALY et al., 2012), e características da empresa focal, que se referem principalmente ao fornecimento, a demanda (JOHNSON, 2001; TOMAS, 2014) e aos aspectos operacionais internos (MANUJ; MENTZER, 2008).

Em relação aos riscos operacionais, pode-se citar riscos com P\&D, planejamento, compartilhamento interfuncional de informações, a estrutura organizacional da empresa, problemas com colaboradores e falhas nos procedimentos administrativos (TOMAS, 2014). Contudo, é incontestável que os riscos na cadeia de suprimentos, na ótica da empresa focal, são voltados ao fornecimento e a demanda (AGUIAR, 2010).

De acordo com Van Landeghem e Vanmaele (2002), os riscos da cadeia de suprimentos relacionados a demanda se referem a incerteza dos clientes, o que gera o efeito-chicote entre os elos da cadeia. Para Manuj e Mentzer (2008), se referem tanto à logística outbound, que gera a probabilidade de os clientes comprarem da empresa focal e à variação no volume e no mix desejados pelo cliente, quanto a falta de confiabilidade nas técnicas de previsão de vendas.

Diabat et al.. (2012) mencionam que a categoria dos riscos relacionados à demanda inclui à movimentação de bens da empresa focal ao cliente, e incluem o risco de obsolescência, excesso ou mesmo falta de estoque. Tomas (2014) complementa os riscos à demanda ao citar as constantes mudanças nas necessidades por parte dos consumidores.

No que tange aos riscos com o fornecimento, as pesquisas de gestão de riscos na cadeia de suprimentos relacionadas ao fornecimento têm sido as mais discutidas do tema, devido a necessidade de se estabelecer estratégias como forma de contenção das principais fontes geradoras de riscos neste lado da cadeia (TOMAS; ALCANTARA, 2013; TOMAS, 2014). Desse modo, os riscos com fornecimento se referem aos resultados relacionados com logística inbound que afeta a habilidade da empresa focal em atender a demanda, gerando gastos, aumento de lead time e ameaças à segurança dos clientes (MANUJ; MENTZER, 2008). 
Diabat et al.. (2012) comentam que a categoria dos riscos com fornecimento aborda a movimentação de materiais de fornecedores à empresa focal, e incluem questões como parcerias com fornecedores, número de fornecedores e (des)centralização com fornecedores. Wagner e Bode (2008) citam outros riscos relacionados ao fornecimento, como a falta de investimento em melhorias de produtos e processos, e a baixa responsividade de fornecedores em atender mudanças repentinas da cadeia.

A falta de confiança no fornecedor, ou conflito de interesses, geram riscos à cadeia (CAMARGO JÚNIOR ET AL., 2014). Há, assim, diversos riscos relacionados ao fornecimento, como paralisações nos fornecedores, não conformidade e baixo desempenho financeiro (TOMAS, 2014). Contudo, fora possível identificar estratégias para mitigação de riscos com o fornecimento, como o desenvolvimento de alianças estratégicas com fornecedores para mitigar o risco do não recebimento dos insumos (FINCH, 2004), ou a adoção de técnicas quantitativas, como a matriz FMEA ou a técnica AHP, para selecionar fornecedores com maior rigidez, de modo a evitar riscos (CAGNIN et al., 2016), ou a coopetição, para que concorrentes desenvolvam cooperação para gestão dos riscos (CAMARGO JÚNIOR et al., 2014).

\section{MODELOS ESTRUTURAIS PARA O SCRM}

Tomas e Alcantara (2013) identificaram inúmeros modelos de gestão de riscos para a cadeia de suprimentos. Aguiar (2010) considera inexistente um modelo universal, que seja confiável a todas as cadeias de suprimentos. As empresas devem, então, analisarem a cadeia de suprimentos que se encontram inseridas, e, então, desenvolverem um modelo de gestão de riscos eficaz nesse sentido.

Independente do modelo de gestão de riscos, Kern et al.. (2012) propõem que os frameworks devem sempre possuir em suas etapas a mitigação dos riscos, sendo esta categoria essencial para o modelo se tornar um fator-chave de competitividade para as empresas. A partir da RSL, foi possível observar que os primeiros modelos para gestão de riscos foram desenvolvidos para a empresa focal, intitulada Processo de Gerenciamento dos Riscos, e consistiam em cinco etapas, sendo elas: I- 
identificação; II- mensuração; III- avaliação; IV- análise quantitativa; V- controle e monitoramento dos riscos (TUMMALA; SCHOENHERR, 2011).

Com relação ao SCRM, o primeiro modelo fora apresentado por Juttner et al. (2003), que consiste em quatro etapas, sendo elas: I- identificação dos riscos; IIdirecionadores dos riscos; III- consequências dos riscos; IV- estratégias de mitigação dos riscos. Ritchie e Brindley (2007) adotaram um modelo similar a este, porém com maior ênfase ao desempenho dos riscos, e conta com cinco etapas: I- origem e perfil dos riscos; II- desempenho dos direcionadores dos riscos; III- desempenho e consequências dos riscos; IV- gestão responsiva dos riscos; V- desempenho e resultado dos riscos.

Ao analisar cadeias de suprimentos envolvendo empresas de grande porte e PME's, Finch (2004) propôs um modelo de gestão de riscos pelo qual a empresa consiga proteger sua posição no mercado, sendo baseado em quatro etapas: Iidentificação dos riscos; II- análise dos riscos; III- mitigação, transferência e aceitação dos riscos; IV- monitoramento dos riscos. Kleindofer e Saad (2005) apontaram a influência de direcionadores do ambiente externo e interno para a incidência de riscos, e apresentaram uma estrutura para sua mitigação, através do modelo SAM (Specifying sources of risk and vulnerabilities, Assessment, and Mitigation), baseado em três níveis, sendo eles a identificação, a avaliação e a mitigação dos riscos.

O modelo de Kern et al. (2012) trata da mitigação dos riscos em quatro etapas, sendo elas: I- identificação dos riscos; II- avaliação dos riscos; III- mitigação dos riscos; IV- desempenho dos riscos, sendo as três primeiras etapas submetidos ao processo de melhoria contínua. No Brasil, o modelo mais recente foi apresentado por Tomas e Alcantara (2013), cujo modelo está apresentado na Figura 5. 
Figura 5 - Modelo estrutural de gestão dos riscos

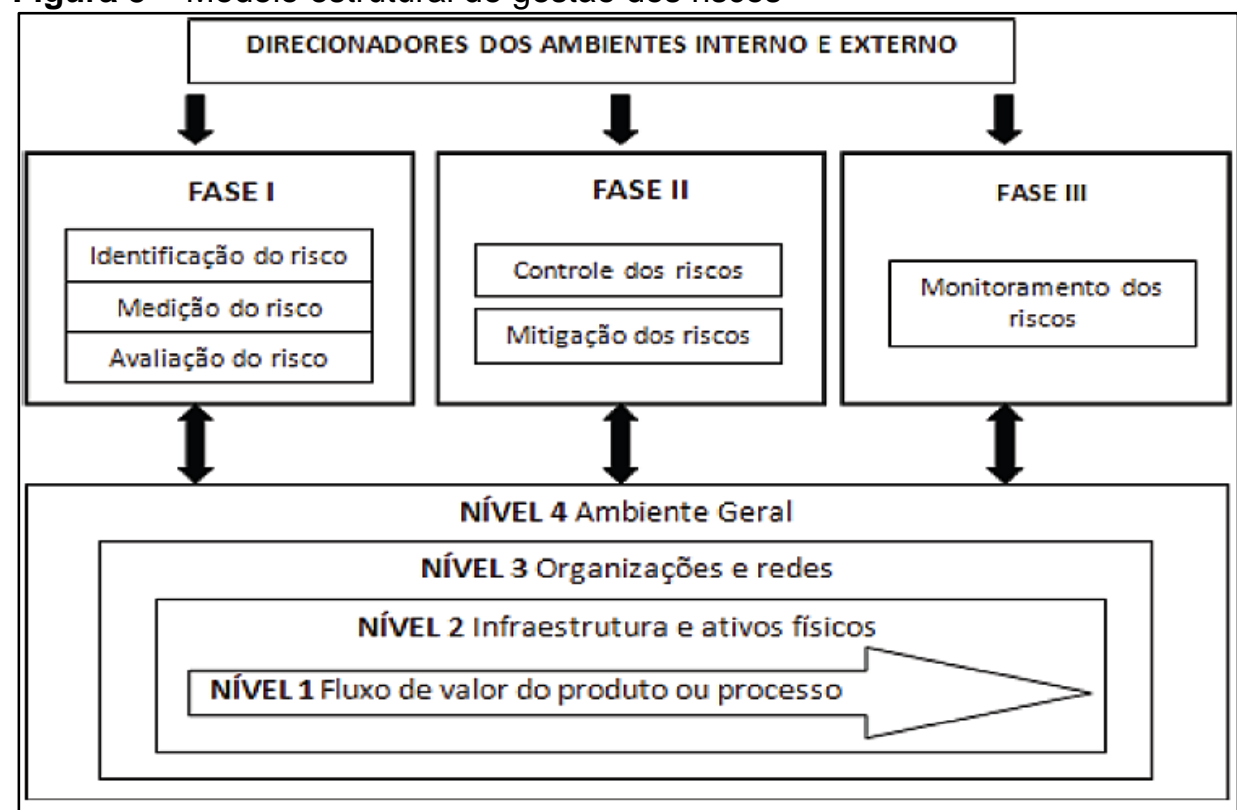

Fonte: Tomas; Alcantara (2013, p. 706).

Outro modelo nacional, voltado a cadeia agroindustrial, foi apresentado por Silva et al. (2017), e conta com 3 fases, sendo elas: I- identificação e categorização dos riscos; II- avaliação dos riscos, com base em análise qualitativa, avaliação de severidade e probabilidade de ocorrência e priorização dos riscos; Ill- mitigação dos riscos, através da identificação teórica das medidas de mitigação efetiva estratégia de mitigação. Com base nos modelos apresentados, foi possível definir uma linha cronológica de cada modelo, considerando a similaridade entre cada framework dos autores, conforme consta na Figura 6.

A partir desta análise cronológica, pode-se observar que todos os modelos apontam a primeira etapa como identificação dos riscos, sendo esta imprescindível para a mitigação dos riscos. Além disso, o processo de avaliação dos riscos também é comumente encontrado na maioria dos modelos, assim como a mitigação ou controle dos riscos. Alguns modelos, contudo, ampliam o horizonte da mitigação dos riscos, ao apresentarem a medição do desempenho como necessária para validar a eficácia do modelo proposto. 
Figura 6 - Linha cronológica dos principais frameworks de SCRM

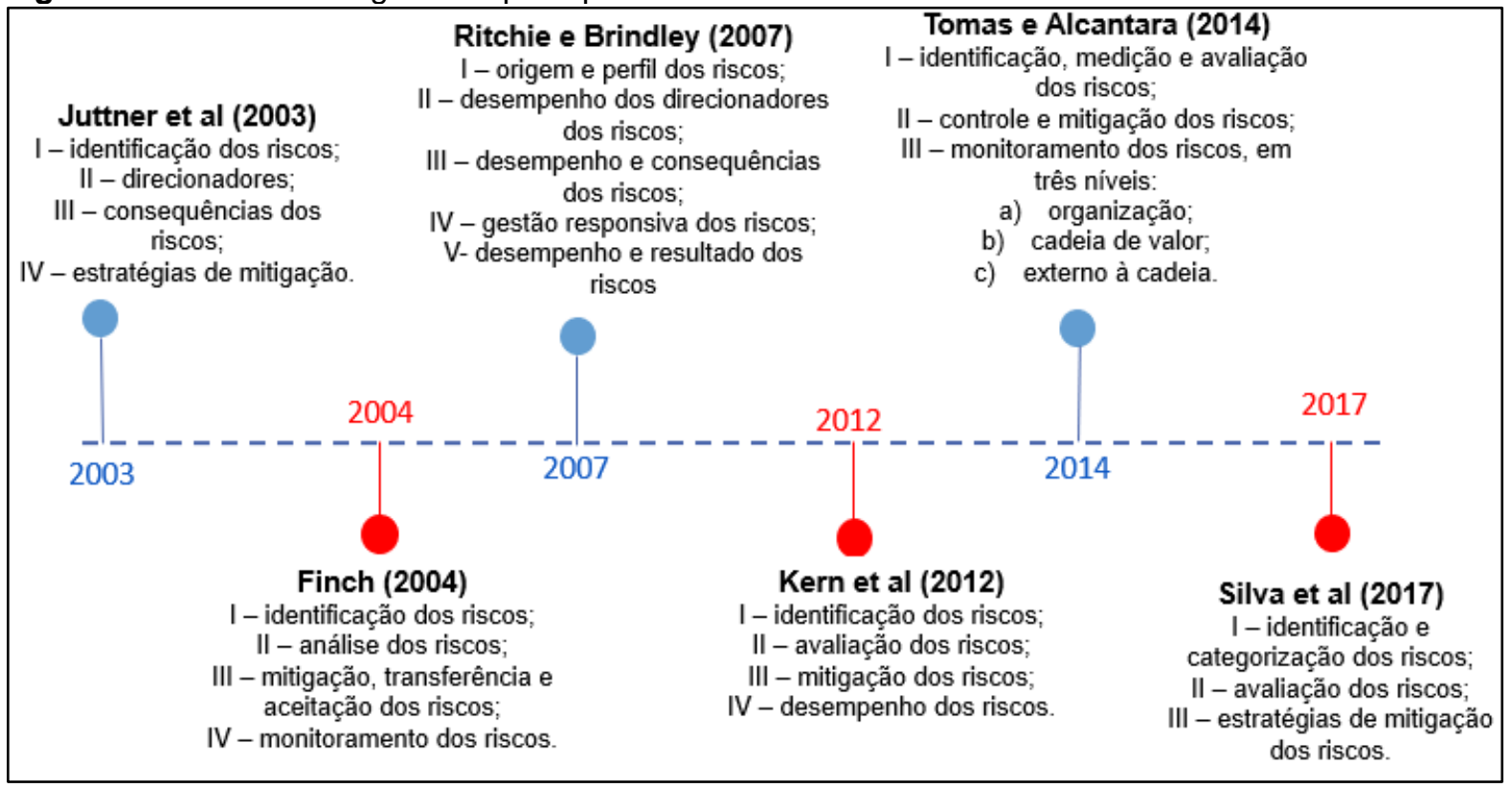

Fonte: Elaborado dos autores (2019)

\section{ESTRATÉGIAS DE MITIGAÇÃO DOS RISCOS E FATORES-CHAVE DO SCRM}

Monroe et al. (2014) observaram que a maioria das pesquisas relacionadas ao tema de SCRM aborda técnicas para mitigação dos riscos, visto a necessidade empresarial de estratégias nesse sentido. Silva et al. (2017) ressaltam a importância da análise crítica dos riscos em todos os elos da cadeia, para então propor estratégias de mitigação. Por sua vez, Vishnu et al. (2019) define que a mitigação dos riscos se refere ao controle e diminuição dos efeitos dos três maiores componentes dos riscos para a cadeia, sendo eles a ruptura na cadeia, a incerteza com o fornecimento e a incerteza com a demanda.

Juttner et al. (2003) apresentaram quatro estratégias de mitigação, sendo elas: evitar; controlar; cooperar; flexibilizar. Chopra e Sodi (2004) apontaram oito estratégias para esse fim: adicionar capacidade; adicionar estoque; elevar base de fornecedores; aprimorar a responsividade; aumentar a flexibilidade; agregar a demanda; aumentar a capabilidade; aumentar a carteira de clientes. Estas estratégias focam principalmente na ótica da empresa focal, voltado a demanda e a base de fornecimento.

Ampliando os horizontes, Manuj e Mentzer (2008) apresentam seis diferentes estratégias para mitigação dos riscos: postponement, especulação; limitar o número 
de fornecedores nos elos da cadeia; controlar, compartilhar e transferir; assegurar; evitar. De acordo com Diabat et al. (2012), algumas técnicas de mitigação aos riscos se referem a: prevenir os riscos através do estudo acerca do tema de SCRM; controlar o impacto do risco, de modo que mesmo caso o risco exista, seu impacto seja mitigado; aumentar o mix de produtos e serviços, entre todos os elos da cadeia. Camargo Júnior et al. (2014) trazem a coopetição como técnicas de mitigação dos riscos. Aqlam e Lam (2015) mencionam que as estratégias de mitigação devem ponderar acerca das origens dos riscos, bem como da intersecção entre si.

É possível perceber inúmeras estratégias de mitigação, o que Monroe et al. (2014) apontam como uma situação negativa, uma vez que quanto maior a gama de estratégias e técnicas de mitigação, mais divergentes as estratégias se tornam. Assim, as estratégias de mitigação podem ser eficazes, caso a empresa reconheça seu segmento, sua cadeia, seus riscos e, então, adote estratégias de mitigação.

\section{CONSIDERAÇÕES E PERSPECTIVAS FUTURAS DO SCRM}

O conceito de SCRM têm aumentado seus debates ao longo dos anos, uma vez que as empresas vêm buscando adotar estratégias considerando todos os elos da cadeia, e os riscos prejudicam a eficiência desta estratégia (COLLICHIA et al., 2012). Ao identificar os principais riscos suscetíveis a cadeia de suprimentos, os principais frameworks para a SCRM, bem como as principais estratégias de mitigação destes riscos, a pesquisa serve como referência tanto à empresas que visam adotar estratégias de SCRM, quanto aos pesquisadores da área, visto a escassez de pesquisas nesse sentido.

Desse modo, sugere-se que trabalhos futuros apresentem técnicas para medição do desempenho dos modelos de gestão de riscos, e possam ainda analisar uma empresa para desenvolver um framework para a mitigação dos riscos da empresa, assim como de toda a cadeia em que ela se encontra.

\section{AGRADECIMENTOS}

Os autores agradecem ao Conselho Nacional de Pesquisa e Desenvolvimento (CNPq), pela bolsa concedida à realização desta pesquisa. 


\section{REFERÊNCIAS}

AGUIAR, E.C. Contribuição ao estudo do fator risco no desempenho de organizações e cadeias de suprimentos. Tese (Doutorado em Administração). Faculdade de Economia, Administração e Contabilidade, Universidade de São Paulo (FEA-USP), 2010.

AQLAN, F.; LAM, S.S. Supply chain risk modelling and mitigation. International Journal of Production Research, 2015. http://dx.doi.org/10.1080/00207543.2015.1047975.

ASSOCIAÇÃO BRASILEIRA DE NORMAS TÉCNICAS (ABNT). NBR ISO 31000:2018: Gestão de Riscos: princípios e diretrizes. Rio de Janeiro, 2018.

BANDALY, D.; SATIR, A.; KAHYAOGLU, Y.; SHANKER, L. Supply chain risk management I: Conceptualization, framework and planning process. Risk Management, v. 14, n. 4, p. 249-271, 2012. https://doi.org/10.1057/rm.2012.7

CAGNIN, F.; OLIVEIRA, M.C.; SIMON, A.T.; HELLENO, A.L.; VENDRAMINI, M.P. Proposal of a method for selecting suppliers considering risk management. International Journal of Quality \& Reliability Management, v. 33, n. 4, 2016. https://doi.org/10.1108/IJQRM-11-2014$\underline{0172}$

CAMARGO JÚNIOR, J.B. de.; VITORINO FILHO, V.A.; PIRES, S.R.I.; SACOMANO NETO, M. Coopetição como estratégia de auxílio na gestão de riscos em cadeias de suprimentos.

Revista Ibero-Americana de Estratégia, v.13, n. 2, 2014.

https://doi.org/10.5585/riae.v13i2.2030

CHRISTOPHER, M.; LEE, H. Mitigating supply chain risk through improved confidence. International Journal of Physical Distribution \& Logistics Management, v. 34, No. 5, 2004, p.388-396. https://doi.org/10.1108/09600030410545436

CHRISTOPHER, M.; PECK, H. Building the resilient supply chain. International Journal of Logistics Management, v. 15, n 2, 2004. https://doi.org/10.1108/09574090410700275

COLICCHIA, C.; STROZZI, F. Supply chain risk management: a new methodology for a systematic literature review. Supply Chain Management: An International Journal, v. 17, n. 4, p. 403-418, 2012. https://doi.org/10.1108/13598541211246558

DIABAT, A.; GOVINDAN, K.; PANICKER, V.V. Panicker Supply chain risk management and its mitigation in a food industry. International Journal of Production Research, 2012. https://doi.org/10.1080/00207543.2011.588619

ENYINDA, C.I. Modeling Enterprise Risk Management in Operations and Supply Chain: A Pharmaceutical Firm Context. Operations and Supply Chain Management, v. 11, n. 1, p. 1-12, 2018. https://doi.org/10.31387/oscm0300195

FAISAL, M.N.; BANWET, D.K.; SHANKAR, R. Quantification of risk mitigation environment of supply chains using graph theory and matrix methods. European Journal Industrial Engineering, v. 1, n. 1, 2007. https://doi.org/10.1504/EJIE.2007.012652

FAWCETT, S. E.; WALLER, M. A.; MILLER, J. W.; SCHWIETERMAN, M. A. HAZEN, B. T.; OVERSTREET, R. E. A trail guide to publishing Success: Tips on Writing Influential Conceptual, Qualitative, and Survey Research. Journal of Business Logistics, v.35, n.1, p.1-16, 2014. https://doi.org/10.1111/jbl.12039 
FINCH, P. Supply chain risk management. Supply Chain Management: An International Journal, v. 9, n. 2, p. 183-196, 2004. https://doi.org/10.1108/13598540410527079

FOLETTO, M. Saiba quais são os serviços afetados pela greve dos caminhoneiros. 0 Globo. Disponível em: https://oglobo.globo.com/economia/ saiba-quais-sao-os-servicosafetados-pela-greve-dos-caminhoneiros-22722891. Acesso em: 31 de maio, 2018.

GONÇALVES, D.P. Principais desastres ambientais no Brasil e no Mundo. 2017. Disponível em: https://www.unicamp.br/unicamp/ju/noticias/2017/12/01/principais-desastresambientais-no-brasil-e-no-mundo. Acesso em: 30 de março, 2019.

HANDFIELD, R.; McCORMACK, K. Supply chain risk management: minimizing disruptions in global sourcing. Taylor \& Francis Group: 134 p., 2008.

https://doi.org/10.1201/9781420013306

JOHNSON, M.E. Learning from toys: lessons in managing supply chain risk from the toy industry. California Management Review, 2001, v. 43, n. 3, 106-124.

https://doi.org/10.2307/41166091

JUTTNER, U.; PECK, H.; CHRISTOPHER, M. Supply chain risk management: Outlining an agenda for future research. International Journal of Logistics: Research \& Applications, v. 6 , n. 4, p. 197-210, 2003. https://doi.org/10.1080/13675560310001627016

KERN, D.; MOSER, R.; HARTMANN, E.; MODER, M. Supply risk management: model development and empirical analysis. International Journal of Physical Distribution \& Logistics Management, v. 42, p. 60 - 82, 2012. https://doi.org/10.1108/09600031211202472

KLEINDORFER, P.R.; SAAD, G.H. Managing Disruption Risks in Supply Chains.

Production and Operations Management, v. 14, n. 1, p. 53-68, 2005.

https://doi.org/10.1111/j.1937-5956.2005.tb00009.x

MANUJ, I.; MENTZER, J.T. Global supply chain risk management strategies. International Journal of Physical Distribution \& Logistics Management, v. 38, n. 3, p.192-223, 2008. https://doi.org/10.1108/09600030810866986

MATOS, A.L.T.; VITORINO FILHO, V.A.; SPERS, V.R.E.; PIRES, S.R.I. A produção acadêmica internacional sobre gestão de riscos na cadeia de suprimentos no período entre 2005 e 2014. Revista de Administração FACES Journal. Belo Horizonte, v.16, n.1, p. 4565, jan./mar, 2017. https://doi.org/10.21714/1984-6975FACES2017V16N1ART3647

MONROE, R.; TEETS, J.; MARTIN, P.R. Supply chain risk management: an analysis of sources of risk and mitigation strategies. International Journal of Applied Management Science, v. 6, n. 1, p. 4-21, 2014. https://doi.org/10.1504/IJAMS.2014.059291

OLIVEIRA, A.C.R. de; FREITAS E SILVA, L.M.; LEITE, M.S.A. Gestão de Riscos em cadeia de suprimentos: aplicação em uma distribuidora de gás canalizado. Revista Produção Online. Florianópolis: v. 18, n. 3, p.1076-1101, 2018. https://doi.org/10.14488/16761901.v18i3.3131

OLIVEIRA, L.G. de.; FREITAS, D.C. de; BATALHA, M.O.; ALCÂNTARA, R.L.C. Gerenciamento de riscos na cadeia agroindustrial de frango: Análise da perspectiva dos 
avicultores em Ubá, Minas Gerais. Revista Produção Online. Florianópolis: v. 15, n. 4, 2015. https://doi.org/10.14488/1676-1901.v15i4.1908

OLIVEIRA, U.R. de; ESPINDOLA, L.S.; MARINS, F.A.S. Perfil de pesquisa sobre gerenciamento de riscos em cadeias de suprimentos. Gestão \& Produção. São Carlos, 2017. https://doi.org/10.1590/0104-530x3515-16

PEDROSO, F.; HOLM-NIELSEN, N. Desastres naturais no Brasil: Um ciclo de tragédias anunciadas. Nexo. Disponível em: https://www.nexojornal.com.br/ensaio/2017/DesastresNaturais-no-Brasil-um-ciclo-de-tragédias-anunciadas. Acesso em: 03 dez., 2018.

PRAKASH, S.; SONI, G.; RATHORE, A.P.S.; SINGH, S. Risk analysis and mitigation for perishable food supply chain: a case of dairy industry. Benchmarking: An International Journal, v. 24, n. 1, p. 2-23, 2017. https://doi.org/10.1108/BIJ-07-2015-0070

RIBEIRO, J. Aumenta vazamento de óleo na Bacia dos Campos. 2011. Exame. Disponível em: https://exame.abril.com.br/mundo/aumenta-vazamento-de-oleo-na-bacia-de-camposinforma-chevron/. Acesso em: 20 mar. 2019. https://doi.org/10.1108/01443570710725563

RITCHIE, B.; BRINDLEY, C. Supply chain risk management and performance: A guiding framework for future development. International Journal of Operations \& Production Management, v. 27, n. 3, p. 303-322, 2007. https://doi.org/10.1080/13675567.2015.1075476

ROGERS, H.; SRIVASTAVA, M.; PAWAR, K.S.; SHAH, J. Supply chain risk management in India - practical insights. International Journal of Logistics Research and Applications: A Leading Journal of Supply Chain Management, 2015.

SENRA, R. Brumadinho, a história de uma tragédia que poderia ter sido evitada. 2019. BBC Brasil. Disponível em: https://www.bbc.com/portuguese/brasil-47399659. Acesso em: 30 de março, 2019.

SHEFFI, Y.; RICE, J. A supply chain view of the resilient enterprise. MIT Sloan Management Review, Fall, 2005, v. 47, n. 1, p.41-48.

SILVA, L.M.F.; OLIVEIRA, A.C.R. de; LEITE, M.S.A.; MARINS, F.A.S. Avaliação do risco na cadeia de suprimento: um estudo exploratório no setor alimentício. Revista Produção Online, Florianópolis, v. 17, n. 1, p. 351-375, 2017. https://doi.org/10.14488/16761901.v17i1.2559

TANG, C.S.; TOMLIN, B. The power of flexibility for mitigating supply chain risks. International Journal of Production Economics, 2008, v. 116, n. 1, p.12-27. https://doi.org/10.1016/.i.jpe.2008.07.008

TALLURI, S.; KULL, T.J.; YILDIZ, H.; YOON, J. Assessing the Efficiency of Risk Mitigation Strategies in Supply Chains. Journal of Business Logistics, v. 34, n. 4, 2013. https://doi.org/10.1111/jbl.12025

TOMAS, R.N.; ALCANTARA, R.L.C. Modelos para gestão de riscos em cadeias de suprimentos: revisão, análise e diretrizes para futuras pesquisas. Gestão \& Produção, v. 20, n. 3, p. 695-712, São Carlos, 2013. https://doi.org/10.1590/S0104-530X2013000300014 
TRANFIELD, D.; DENYER, D.; SMART, P. Towards a Methodology for Developing Evidence-Informed Management Knowledge by Means of Systematic Review. British Journal of Management, v. 14, 2003. https://doi.org/10.1111/1467-8551.00375

TUMMALA, R.; SCHOENHERR, T. Assessing and managing risks using the Supply Chain Risk Management Process (SCRMP). Suppl Chain Management - Na International Journal, v. 16, n. 6, p. 474-483, 2011. https://doi.org/10.1108/135985411111171165

VAN LANDEGHEM, H.; VANMAELE, H. Robust planning: a new paradigm for demand chain planning. Journal of Operations Management, v. 20, p. 769-783, 2002. https://doi.org/10.1016/S0272-6963(02)00039-6

VENTURA, L.A.S. Incêndio contaminou água e matou peixes em Santos. Exame. 2015. Disponível em: https://exame.abril.com.br/brasil/incendio-contaminou-agua-em-santos-dizrelatorio/. Acesso em: 30 de março, 2019.

VILKO, J.; HALLIKAS, J. Origin and impact of supply chain risks affecting supply security. International Journal of Shipping and Transport Logistics, v. 4, n. 2, 2012. https://doi.org/10.1504/IJSTL.2012.046190

VISHNU, C.R; SRIDHARAN, R.; RAM KUMAR, P.N. Supply chain risk management: models and methods. International Journal of Management and Decision Making, v. 18, n. 1, 2019. https://doi.org/10.1504/IJMDM.2019.10013542

WAGNER, S.M.; BODE, C. An empirical examination of supply chain performance along several dimensions of risk. Journal of Business Logistics, v. 29, n. 1, 2008. https://doi.org/10.1002/j.2158-1592.2008.tb00081.x

WAGNER, S.M.; NESHAT, N. Assessing the vulnerability of supply chains using graph theory. International Journal of Production Economics, p. 121-129, 2010. https://doi.org/10.1016/j.ijpe.2009.10.007

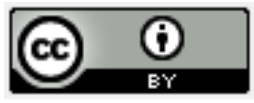

Artigo recebido em: 06/04/2019 e aceito para publicação em: 16/08/2019 DOI: http://dx.doi.org/10.14488/1676-1901.v19i3.3605 